\title{
INFLUENCIA DE LA AUTONOMÍA DEL APRENDIZAJE EN LA CALIDAD DE LA FORMACIÓN PROFESIONAL EN LA UNMSM
}

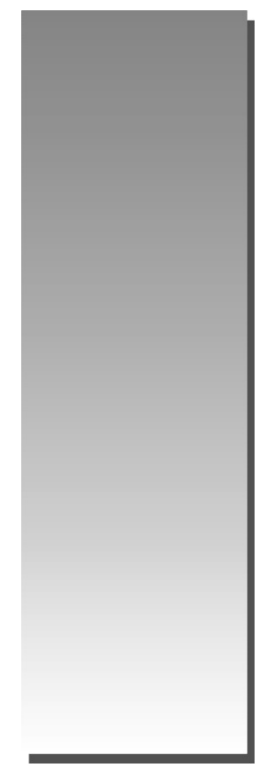

Teresa Ríos Delgado*

transatelier@yahoo.com

\begin{abstract}
RESUMEN
La fundamentación de la autonomía del aprendizaje se inicia con las diferentes teorías de aprendizaje - constructivismo, cognitivismo, conductista, etc. El concepto de la autonomía del aprendizaje se puede apreciar desde diversos autores y contextos. El aprendizaje autónomo se presenta en base a las condiciones, características, estrategias y técnicas para su desarrollo asimismo como el rol del docente universitario. El objetivo de la presente investigación es contribuir con un modelo de aprendizaje autónomo en la calidad de la formación profesional del egresado de la Universidad Nacional Mayor de San Marcos. El método utilizado es el ex pos facto, teniendo como participantes al quinto superior de las Facultades de Ciencias Administrativas, Derecho y Ciencia Política, y Educación. En Instrumentos de recolección de datos se diseñó una lista de cotejo. Como conclusión de la investigación, se acepta la hipótesis general que el estilo de aprendizaje autónomo contribuye a elevar la calidad de la formación profesional de los alumnos de los últimos semestres de la UNMSM.

Palabras claves: autonomía del aprendizaje, aprendizaje autónomo, calidad de la formación Profesional.
\end{abstract}

\begin{abstract}
The basis of the autonomy of the learning begins with the different learning theories - constructivism, cognitive, behaviorist, etc. The concept of the autonomy of the learning can be appreciated from diverse authors and contexts. The autonomous learning is showed up based on the conditions, characteristics, strategies and techniques for its development as well as the faculty's role. The objective of the present research is to contribute with a model of autonomous learning in the quality of the professional formation of the graduate of San Marcos University. The used method is the ex pos facto, having as participants to the fifth superior of the Faculties of Administrative Sciences, Education, Medicine and Law and Political Science. In Instruments of gathering of data a check off list of survey was designed. As conclusion of this research, the general hypothesis established that the Style of Autonomous Learning contributes to elevate the Quality of the Professional Formation of the students of the last semesters of UNMSM is accepted.
\end{abstract}

Keywords: autonomy of the learning, autonomous learning, quality of the professional formation.

\footnotetext{
* Doctorado en Educación, traductora, Docente de la Unidad de Postgrado de la Facultad de Ciencias Administrativas de la UNMSM, Docente Investigadora.
} 


\section{INTRODUCCIÓN}

Nos encontramos en la nueva educación, con un docente tutor, guía y facilitador y un estudiante, creador e investigador. El aprendizaje a nivel superior debe realizarse en base a las leyes del aprendizaje del adulto. Es así que siendo el estudiante el núcleo de esta institución superior, el trabajo del estudiante se debe establecer como sujeto activo del aprendizaje, y en concordancia con los demás miembros de la universidad - docentes, autoridades, etc. En nuestro contexto, nivel universitario, debemos centrarnos en la formación del adulto que requiere de una concepción de enseñanza que promueva el proceso de aprendizaje autónomo, es decir un estilo de aprendizaje que le conduzca a lograr todos sus objetivos en su formación profesional. El niño tiene una percepción global de todo lo que se le enseña, en cambio el adulto tiene una percepción selectiva, es decir es discriminador y despliega su autonomía cuando selecciona lo que le gusta, lo que le brinda ventajas, percibe la realidad de forma selectiva, posee autonomía personal.

La calidad de la formación profesional por lo general se considera un termómetro del desempeño del estudiante a lo largo de su vida universitaria. Sin embargo, lograr una correspondencia de igualdad semántica entre ambos términos se podría lograr si se mejora los diversos factores que implica el desarrollo de este aprendizaje. La sociedad exige mantener altos estándares de calidad, lo que obliga a diseñar currículos y sistemas educativos acordes con esta nueva realidad. Existen diversos factores que influyen en la calidad de la formación profesional, pero en la presente investigación centraremos nuestra atención en: el aprendizaje autónomo.

La universidad debe formar profesionales intelectuales suficientes para enfrentarse a la incertidumbre del desarrollo acelerado de la información además de ser capaces de crear sus propias herramientas de trabajo con las que van a dar sentido y eficacia a ese conocimiento cambiante y mutante. A esta capacidad de creación autónoma de herramientas la denominamos aprendizaje autónomo. Así, una persona autónoma es "aquella cuyo sistema de autorregulación funciona de modo que le permite satisfacer exitosamente tanto las demandas internas como externas que se le plantean, (Bornas, 1994).

La literatura consultada, específicamente las obras de Monereo y de otros destacados investi- gadores, nos ofrece resultados de investigaciones y reflexiones teóricas a la luz de experiencias prácticas en diversos contextos educativos para la incorporación de las estrategias de aprendizaje en el diseño curricular de enseñanza básica y secundaria. Sin embargo, muy poco encontramos a nivel superior, específicamente a nivel universitario. Empero, nuestra experiencia como docente y estudiante universitario es fundamental en el desarrollo de nuestra propuesta.

Nuestra preocupación está centrada pues en identificar la contribución que inciden en la calidad de la formación profesional.

\section{MATERIALES Y MÉTODOS}

En la investigación se aplicó el muestreo probabilístico (Munch y Ángeles, 2005), partiendo del supuesto de que cada elemento de la población tiene la misma probabilidad de ser seleccionado en la muestra. La población estuvo constituida por la totalidad de los alumnos egresados en el año 2007 de las Facultades de Ciencias Administrativas, Derecho, Educación y Medicina de la Universidad Nacional Mayor de San Marcos, que hacen un total de 1157. Los datos se han tomado de la Oficina de Matricula y/o estadística de las cuatro facultades respectivas. La población objetivo fue seleccionada en base a los alumnos que pertenecieron al quinto superior (marco muestral), que sumaron 231 alumnos. Como el marco muestral ha sido construido en base a diversos registros de las facultades, por lo tanto estos datos son estadísticos probabilísticos ya que establecen el $20 \%$ del total de egresados de las cuatro facultades consideramos que el tamaño de muestra más adecuado, para nuestra investigación, era la obtenida mediante la fórmula de la población infinita, la que arrojó $\mathrm{n}=83$ alumnos.

$\mathrm{n}=(\mathrm{Z})^{2} \mathrm{p} \cdot \mathrm{q}$

$\mathrm{E}^{2}$

$\mathrm{Z}=1.96 ; \mathrm{p}=\mathrm{q}=0.5 ; \mathrm{E}=0.055$.

Donde $\mathrm{n}=82.6 \equiv 83$ alumnos

Para el estudio de la variable de Autonomía del aprendizaje, se elaboró una tabla de cotejo que permitió verificar si efectivamente se aplica en los alumnos del quinto superior egresados. Para el estudio de la variable de Calidad de la Formación Profesional, se trabajó con los estudiantes que pertenecieron al quinto superior que permitió verificar si la calidad es baja o alta. 


\section{RESULTADOS}

Se han relacionado las dos variables cualitativas, estilo de aprendizaje autónomo y calidad de la formación profesional, utilizando la prueba estadística de la Chi Cuadrado, que permite obtener las correlaciones de Pearson y de Spearman, según se trate de variables cuantitativas o cualitativas, respectivamente. La tabla de contingencia se construye con los datos proporcionados por los estudiantes encuestados.
Tabla de contingencia Calidad * Aprendizaje Recuento

\begin{tabular}{|l|l|c|c|c|}
\hline \multicolumn{2}{|l|}{} & \multicolumn{2}{|c|}{ Aprendizaje } & Total \\
\hline \multicolumn{2}{|l|}{} & $\mathrm{Si}$ & No & $\mathrm{Si}$ \\
\hline Calidad & $\mathrm{Si}$ & 75 & 2 & 77 \\
\hline F.Prof. & $\mathrm{No}$ & 4 & 2 & 6 \\
\hline \multicolumn{2}{|l|}{ Total } & 79 & 4 & 83 \\
\hline
\end{tabular}

El Valor $\mathrm{p}=0.001<0.05$

Pruebas de chi-cuadrado

\begin{tabular}{|l|c|c|c|c|c|}
\hline & Valor & gl & $\begin{array}{c}\text { Valor } p \\
\text { (bilateral) }\end{array}$ & $\begin{array}{c}\text { Valor } p \\
\text { (bilateral) }\end{array}$ & $\begin{array}{c}\text { Valor } p \\
\text { (unilateral) }\end{array}$ \\
\hline Chi-cuadrado de Pearson & $11,464(\mathrm{~b})$ & 1 &, 001 & & \\
\hline $\begin{array}{l}\text { Corrección por } \\
\text { continuidad(a) }\end{array}$ & 5,742 & 1 &, 017 &, 025 &, 025 \\
\hline Razón de verosimilitudes & 5,876 & 1 &, 015 & & \\
\hline $\begin{array}{l}\text { Estadístico exacto de } \\
\text { Fisher }\end{array}$ & & 1 &, 001 & & \\
\hline Asociación lineal por lineal & 11,326 & 1 & & & \\
\hline N de casos válidos & 83 & & & & \\
\hline
\end{tabular}

\section{Interpretación}

Con el valor $\mathrm{p}=0.001<0.05$, podemos afirmar que el estilo de aprendizaje autónomo contribuye a elevar la calidad de la formación profesional de los alumnos de los últimos semestres de la UNMSM. La relación de las variables es directa y significativa, y alcanza una correlación de Nivel Aceptable de $37.2 \%$.

\section{DISCUSIÓN}

Debemos situarnos en un contexto de educación superior, en nuestro caso la educación en la universidad por que hablar de autonomía nos lleva a hablar del estudiante que puede ser independiente en la creación y construcción de sus conocimientos, y uno de los contextos donde se desarrolla este fenómeno es la universidad. Por tanto, el estudiante universitario debe percibir y percibe el aprendizaje como un andamiaje conceptual conectado a sus objetivos y metas

Lamentablemente en nuestro medio uno de los aspectos que más genera desventajas en los estudiantes universitarios es la deficiencia auto- nomía e independencia en período de educación básica y media, sin haber aprendido estrategias que los ayuden en su andamiaje cognitivo. En general las destrezas y/o habilidades en el estudio han sido producto de un ensayo y error en sus técnicas de estudio. Y esto se puede observar en los estudiantes universitarios de los primeros ciclos de la universidad: inician sus estudios con mucha motivación pero no se les ha enseñado a estudiar. Y lo experimentado como docente en estos años de cátedra a los ingresantes fundamenta este enunciado.

Esta carencia de un método de estudio desarrolla aprendizajes memorísticos, y la memoria a corto plazo es el almacén pusilánime de los constructos y se ha dado casos en que los estudiantes no llegan a comprender realmente lo que están estudiando; $y$ encontramos estudiantes que fracasan en sus aprendizajes aún estudiando con esfuerzo y voluntad. El manejo de una técnica apropiada conduce al logro de aprendizajes significativos que permiten relacionar, reflexionar, jerarquizar, seleccionar y participar activamente en el proceso de estudio logrando una cierta organización, 
orden y metodología para abordar los contenidos de su carrera profesional.

Hemos sostenido que la educación es un proceso que involucra a docentes, estudiantes y al medio, entonces un aprendizaje exitoso requiere aparte de la habilidad intelectual, el compromiso con la tarea, y la producción creativa del conocimiento, pero sobre la interacción de estos tres componentes.

Es cierto que el problema de investigación se refiere a varios niveles o dimensiones que incluyen el proceso de enseñanza impartida por los docentes. Empero, en la investigación realizada se delimitó sólo trabajar con la perspectiva del aprendizaje que tiene los alumnos. Ello se debe a dos razones complementarias: la primera es que se delimitan los alcances y limites del problema dentro de la óptica del proceso de aprendizaje, es decir, desde la óptica de los estudiantes egresados y no desde la perspectiva de los docentes pese a que también esta última es importante.

Nuestra propuesta en base a los resultados del presente trabajo es una propuesta del desarrollo de una didáctica universitaria que junto a un currículo de contenidos equilibren la concepción de la educación universitaria por la importancia en la formación.

\section{CONCLUSIONES}

1. El estilo de aprendizaje autónomo contribuye a elevar la calidad de la formación profesional de los estudiantes de los últimos semestres de la UNMSM

2. Los estudiantes necesitan aprender a aprender como vehículo esencial para adquirir conocimientos autónomamente, en medio de las estructuras de aprendizaje impuestas por el marco institucional en el cual participan.

3. En términos prácticos, se ha observado reiteradamente, que cuando se potencia la autonomía el estudiante cambia la interpretación de los distintos elementos y procesos curriculares, tanto para los estudiantes como para los docentes

4. Hemos comprobado que la docencia centrada en el estudiante, requiere capacitarlo para el aprendizaje autónomo y dotarlo de herramientas para el estudio.
5. Los materiales didácticos pasan a convertirse en recursos capaces de generar conocimientos de alto nivel y de facilitar el aprendizaje autónomo. Un importante papel juegan, en este sentido, las nuevas tecnologías de la información y la comunicación.

6. Ser capaces de aprender autónomamente es una garantía de desarrollo personal, mayor espacio de libertad interior y exterior, y mejor adaptación a un ambiente en continuo cambio.

\section{LITERATURA CITADA}

APARICIO IZQUIERDO, F. (1994) La calidad de la enseñanza superior y otros temas universitarios. Madrid, Editorial ICE de la Universidad Politécnica.

BORNAS, X (1994) La autonomía personal en la infancia. Estrategias cognitivas y pautas para su desarrollo. España, Siglo XXI editores

DELORS, J (1996) La educación encierra un tesoro. Ed. Santillana. UNESCO Madrid.

GARCÍA, A y otros (2001) Didáctica universitaria. La Muralla. Madrid.

HARVEY Lee (1998) " La Educación en el siglo XXI" . en : Calidad de la Educación, una publicación del Consejo Superior de Educación (CSE), Chile. Diciembre.

HERNÁNDEZ Pina, F y otros (2005) Aprendizaje, competencias y rendimiento en la Educación Superior. La Muralla S A. Madrid.

KAPSOLI , W (1999) Ricardo Palma: Una Universidad en construcción, Lima Ed. Universidad Ricardo Palma

Marzano (1992): Citado por INSUATY, Luis Delfín. Especialización en Pedagogía para el Desarrollo del Aprendizaje Autónomo. UNADCAFAM. Módulo A.

Monereo, C. y Pozo, J. (2005). La universidad ante la nueva cultura. Enseñar y aprender para la autonomía. Madrid: Santillana.

MONEREO, C y CASTELLO, M (1997) Las estrategias de aprendizaje. Cómo incorporarlas a la práctica educativa, Barcelona, Edebé.

MONEREO, C y BARBERA, E (2000) "Diseño instruccional de las estrategias de aprendizaje en entornos educativos no-formales". En 
Monereo et al. Estrategias de aprendizaje. Madrid, Visor/Ediciones de la Universitat Oberta de Catalunya.

MUÑOZ I, VILLA L., L. y MÁRQUEZ J., A. Calidad de la Educación: Políticas instrumentadas en diversos países para mejorarla, México: Universidad Iberoamericana/Instituto de Fomento e Investigación Educativa.

POZO, I. (2000) Aprendices y maestros, la nueva cultura de aprendizaje. Ed. Alianza .Madrid.

RAMOS, G. (1999) La Universidad peruana en el siglo XXI, Lima, Ed. Universidad Ricardo Palma.

STENHOUSE, L. (1987): La investigación como base de la enseñanza. Madrid: Morata

TRIM, J.J.M. (1988): "Preface”. En H.Holec (ed): Autonomy and self-directed learning: present fields of application. Strasbourg: Council for Cultural Cooperation, Council of Europe.

VINCENC B. y otros. (1995) La formación universitaria a debate. Barcelona.
ZABALZA, Miguel A. (2004) La Docencia en el nuevo marco de las enseñanzas universitarias. Universidad de Santiago de Compostela. España

Bibliografía referida a la metodología de la investigación

BISQUERRA, R. (Coord.). (2004). Metodología de la investigación educativa. Madrid: La Muralla.

CEA, D 'ANCONA, Ma. Ángeles (1998) Metodología cuantitativa. Estrategias y técnicas de investigación social. Madrid, España.

HERNÁNDEZ, R.; FERNÁNDEZ, C. y BAPTISTA, P. (2006). Metodología de la investigación. 4a . Edición. México: McGraw Hill. Moreno, M.G. (1986). Introducción a la metodología de la investigación educativa. Guadalajara, México: Progreso.

MUNCH LOURDES y ANGELES ERNESTO, (2005): Métodos y Técnicas de Investigación, Editorial Trillas, 13a Edición, Méjico. 